\title{
MASSIVE STAR FORMATION OF THE SGR A EAST H II REGIONS NEAR THE GALACTIC CENTER
}

\author{
F. Yusef-Zadeh ${ }^{1,8}$, J. H. Lacy ${ }^{2,8}$, M. Wardle ${ }^{3}$, B. Whitney ${ }^{4}$, H. Bushouse ${ }^{5}$, D. A. Roberts ${ }^{6}$, And R. G. ArendT ${ }^{7}$ \\ ${ }^{1}$ Department of Physics and Astronomy, Northwestern University, Evanston, IL 60208, USA \\ ${ }^{2}$ Department of Astronomy, University of Texas, Austin, TX 78712, USA \\ ${ }^{3}$ Department of Physics and Astronomy, Macquarie University, Sydney, NSW 2109, Australia \\ ${ }^{4}$ Space Science Institute, 4750 Walnut Street, Suite 205, Boulder, CO 80301, USA \\ 5 STScI, 3700 San Martin Drive, Baltimore, MD 21218, USA \\ ${ }^{6}$ Adler Planetarium and Astronomy Museum, 1300 South Lake Shore Drive, Chicago, IL 60605, USA \\ ${ }^{7}$ University of Maryland, Baltimore County, GSFC, Code 665, Greenbelt, MD 20771, USA \\ Received 2010 July 13; accepted 2010 October 9; published 2010 November 29
}

\begin{abstract}
A group of four compact $\mathrm{H}$ II regions associated with the well-known $50 \mathrm{~km} \mathrm{~s}^{-1}$ molecular cloud is the closest site of on-going star formation to the dynamical center of the Galaxy, at a projected distance of $\sim 6$ pc. We present a study of ionized gas based on the [Ne II] $(12.8 \mu \mathrm{m})$ line, as well as multi-frequency radio continuum, Hubble Space Telescope $\mathrm{Pa} \alpha$, and Spitzer Infrared Array Camera observations of the most compact member of the H II group, Sgr A East HiI D. The radio continuum image at $6 \mathrm{~cm}$ shows that this source breaks up into two equally bright ionized features, D1 and D2. The spectral energy distribution of the D source is consistent with it being due to a $25 \pm 3 M_{\odot}$ star with a luminosity of $8 \pm 3 \times 10^{4} L_{\odot}$. The inferred mass, effective temperature of the UV source, and the ionization rate are compatible with a young O9-B0 star. The ionized features D1 and D2 are considered to be ionized by UV radiation collimated by an accretion disk. We consider that the central massive star photoevaporates its circumstellar disk on a timescale of $3 \times 10^{4}$ years giving a mass flux $\sim 3 \times 10^{-5} M_{\odot} \mathrm{yr}^{-1}$ and producing the ionized material in D1 and D2 expanding in an inhomogeneous medium. The ionized gas kinematics, as traced by the [Ne II] emission, is difficult to interpret, but it could be explained by the interaction of a bipolar jet with surrounding gas along with what appears to be a conical wall of lower velocity gas. The other H iI regions, Sgr A East A-C, have morphologies and kinematics that more closely resemble cometary flows seen in other compact H II regions, where gas moves along a paraboloidal surface formed by the interaction of a stellar wind with a molecular cloud.
\end{abstract}

Key words: Galaxy: center - H II regions - stars: early-type

\section{INTRODUCTION}

The Galactic center region is known to have a high concentration of massive, warm, dense, and turbulent molecular clouds. Due to strong tidal forces exerted by the gravitational potential of the nuclear cluster and the massive black hole, Sgr A*, only dense molecular gas is expected to survive in this region (see Morris \& Serabyn 1996). This has the ramification that the formation of massive stellar clusters must be pervasive in this region, as evidenced by the Arches and Quintuplet clusters as well as the nuclear massive cluster centered on Sgr A* (Figer et al. 2002; Bartko et al. 2009, and references therein). There are also young clusters of O-B stars traced by ultracompact (UC) H II regions in Sgr B2 (e.g., DePree et al. 1998) which is possibly the best example of mini-starburst activity in the Galaxy. Although our present understanding of star formation is primarily limited to observations of low-mass stars (Shu et al. 1987; McKee \& Ostriker 2007; Zinnecker \& Yorke 2007), probing star-forming sites in the Galactic center region can be useful in addressing the key mechanism by which massive stars are formed $\left(M>10 M_{\odot}\right.$; see Hoare et al. 2007, and references cited therein).

Here, we examine the nature of a group of massive young stars lying within 2.5 of the dynamical center of the Galaxy (Reid \& Brunthaler 2004; Ghez et al. 2008; Gillessen et al. 2009). At a projected distance of $6 \mathrm{pc}$ from $\mathrm{Sgr} \mathrm{A}^{*}$, there is a cluster of compact $\mathrm{H}$ II regions on the edge of the $50 \mathrm{~km} \mathrm{~s}^{-1}$

\footnotetext{
8 Visiting Astronomer at the Infrared Telescope Facility, which is operated by the University of Hawaii under Cooperative Agreement no. NNX-08AE38A with the National Aeronautics and Space Administration, Science Mission Directorate, Planetary Astronomy Program.
}

molecular cloud M-0.02-0.07. This cluster consists of brightest $\mathrm{H}$ II regions in the Galactic center with the exception of the cluster of $\mathrm{H}$ II regions in Sgr B2. The $50 \mathrm{~km} \mathrm{~s}^{-1}$ molecular cloud itself is interacting with the nonthermal Sgr A East (e.g., Tsuboi et al. 2009, and references cited therein) which is known to be a supernova remnant (SNR G0.0+0.0). Since Sgr A East appears to be interacting with the Galactic center circumnuclear molecular ring, which itself orbits $\mathrm{Sgr} \mathrm{A}^{*}$, it is probable that Sgr A East, M-0.02-0.07, and the cluster of $\mathrm{H}$ II regions all lie near the Galactic center, not far from their projected distances. The compact Hil regions, known as the Sgr A East H II A-D (Sgr A A-D), trace the site of recent massive star formation nearest to the dynamical center of the Galaxy. The four H II regions have radial velocities ranging between 43 and $49 \mathrm{~km} \mathrm{~s}^{-1}$, and thus appear dynamically coupled to the $50 \mathrm{~km} \mathrm{~s}^{-1}$ molecular cloud (Goss et al. 1985; Serabyn et al. 1992). With the exception of [Ar III] emission from Sgr A-D H II source, IR spectroscopic measurements detected [Ne II] and [ArIII] line emission from all four components (A-D), indicating that the exciting stars have spectral types of O8-B0 (Serabyn et al. 1992), consistent with $\mathrm{H} 76 \alpha$ measurements (Goss et al. 1985).

The measured extinction toward Sgr A East A-C is less than that of source D (Cotera et al. 1999; Serabyn et al. 1992). Additional extinction for source $\mathrm{D}$ is likely due to the $50 \mathrm{~km} \mathrm{~s}^{-1}$ molecular clouds or due to material associated with the ionizing stellar source (Serabyn et al. 1992). These authors estimate that the extinction values at $12.8 \mu \mathrm{m}$ are $\sim 1-1.3 \mathrm{mag}$ for $\mathrm{A}-\mathrm{C}$ and $\sim 3.3$ toward the Sgr A D source. These extinction measurements imply that the Sgr A East A-C H II regions, which are resolved spatially, are located on the front side of the molecular cloud, whereas the compact Sgr A D source 
or G359.96-0.08 (hereafter this source is called D) may be embedded within the $50 \mathrm{~km} \mathrm{~s}^{-1}$ molecular cloud. Near-IR measurements have also examined the stellar sources associated with these H II regions (Cotera et al. 1999). A highly reddened source with a $H-K^{\prime} \sim 3.5$ is detected at the position of the most compact H II source D. The low fractional abundance of $\mathrm{Ar}^{++}$ led to the suggestion that stellar temperatures of the Sgr A East $\mathrm{H}$ II regions range between 34,500 and 36,500 K (Serabyn et al. 1992) which are consistent with spectra types O8-O9 stars (Goss et al. 1985; Yusef-Zadeh et al. 1989). The spectral identification of the Sgr A D source has not been clear but Cotera et al. (1999) suggest that its spectrum could be consistent with a combination of a $\mathrm{UC} \mathrm{H}$ II region and $\mathrm{B}[\mathrm{e}]$ stars. Here, we investigate radio and infrared properties of the most compact (D source) and extended (A-C sources) of the Sgr A East H II regions.

\section{OBSERVATIONS}

\subsection{Radio Continuum}

Radio continuum observations were made with the D configuration of the Very Large Array (VLA) of the National Radio Astronomy Observatory ${ }^{9}$ (NRAO), at $43 \mathrm{GHz}(7 \mathrm{~mm})$. The source was observed as part of a survey of UC H II regions in the Galactic center, which took place on 2009 May 29. The source was observed in the fast switching mode of the VLA (each cycle had $150 \mathrm{~s}$ on the source and $15 \mathrm{~s}$ on the complex gain calibrator-NRAO530) for a total of 20 minutes. 3C286 was used as a flux calibrator. Standard data editing and calibration of the gains was done in AIPS. Using standard calibration, we also reduced multi-configuration radio continuum data at $8.3 \mathrm{GHz}$ (AY43). The $4.8 \mathrm{GHz}$ data were taken from Yusef-Zadeh \& Wardle (1993).

\section{2. [Ne II] Line and $12.8 \mu \mathrm{m}$ Continuum}

The Sgr A East HiI region cluster was observed in the [Ne II] $(12.8 \mu \mathrm{m})$ line with the high-resolution mid-infrared spectrograph TEXES on the NASA Infrared Telescope Facility (IRTF) on 2009 June 3 and 2010 May 30 (UT). TEXES (Lacy et al. 2002) has several operating modes. These observations were made in two modes: high-resolution, cross-dispersed, with velocity resolution $\sim 4 \mathrm{~km} \mathrm{~s}^{-1}$ and spatial resolution $\sim 1^{\prime \prime}$. 2 along a $1^{\prime \prime} .4 \times 7^{\prime \prime} .5$ slit, and medium-resolution, long-slit, with velocity resolution $\sim 25 \mathrm{~km} \mathrm{~s}^{-1}$ along a $1^{\prime \prime} .4 \times 55^{\prime \prime}$ slit. The pixel spacing is 0 '.36 spatially and $0.95 \mathrm{~km} \mathrm{~s}^{-1}$ spectrally in cross-dispersed mode. It is 0.36 and $8.9 \mathrm{~km} \mathrm{~s}^{-1}$ in long-slit mode. Maps were made by stepping the $\mathrm{N}-\mathrm{S}$ oriented slit to the east, with $00^{\prime \prime} 7$ steps in 2009 and 0.35 steps in 2010. The Sgr A East H II region was mapped in 2009 in cross-dispersed mode by making a series of scans, each covering $30^{\prime \prime} \times 7^{\prime \prime} .5$ (R.A. $\times$ decl.), which were pieced together to cover a $34^{\prime \prime} \times 68^{\prime \prime}$ region (with some small gaps), with the help of a long-slit scan that covered the entire region. H II source D was reobserved in 2010 to obtain better spatial sampling and higher signal-to-noise ratio. These measurements also allowed us to detect $12.8 \mu \mathrm{m}$ emission from the D source using our low spectral resolution data and mediumresolution data taken in 2009.

The spectral coverage of the data was $2270 \mathrm{~km} \mathrm{~s}^{-1}$, which in the cross-dispersed observations was broken into eight spectral

\footnotetext{
9 The National Radio Astronomy Observatory is a facility of the National Science Foundation, operated under a cooperative agreement by Associated Universities, Inc.
}

orders of the high-resolution grating. [Ne II] line emission was detected in a $60 \mathrm{~km} \mathrm{~s}^{-1}$ interval centered at $v_{\mathrm{LSR}} \approx 46 \mathrm{~km} \mathrm{~s}^{-1}$. Dust continuum emission was only marginally detected outside of this spectral interval (also see Figure 3 showing source D before and after deconvolution.)

The data were first processed with the standard TEXES pipeline reduction program (Lacy et al. 2002), which removes spikes, corrects for optical distortions, and produces wavelength and intensity calibrated maps. It was then processed with a maximum-entropy-method deconvolution routine, which improved the spatial resolution to about 0.7 , slightly better than the IRTF diffraction limit. Because a part of the point-spread function (PSF) is caused by seeing, which cannot be exactly the same for the observations of the PSF calibrator (VX Sgr) and the $\mathrm{H}$ II region, the results of the deconvolution should be viewed with some caution, but the close similarity between the deconvolved [ $\mathrm{Ne}$ II] data and the higher resolution radio continuum data indicates that the results should be reliable. In addition, all of the features that we discuss in the [Ne II] data can be seen before deconvolution, although some sources, notably the two peaks in H II source D, blend together. The absolute astrometry of the $12.8 \mu \mathrm{m}$ data is about few arcseconds. We obtained coordinates for the $[\mathrm{Ne}$ II] maps by aligning them with the radio maps. These should be good to better than 0'.35 (1 pixel). The relative astrometry of the [Ne II] and $12.8 \mu \mathrm{m}$ continuum should be better since they were observed simultaneously.

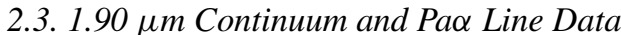

$\mathrm{Pa} \alpha$ emission-line and continuum images of the Sgr A East region were obtained as part of the larger survey of the Galactic center by Wang et al. (2010), using the Near-Infrared Camera and Multi-Object Spectrometer (NICMOS) on the Hubble Space Telescope (HST) (program 11120). Exposures were obtained in March-May 2008 using the NICMOS camera 3, which has a pixel scale of 0.20 . Images were obtained in narrowband filters centered on the $\mathrm{Pa} \alpha$ emission line at $1.87 \mu \mathrm{m}$ and the nearby continuum at $1.90 \mu \mathrm{m}$. A pattern of dithered observations was obtained at each pointing in the survey with each exposure having a duration of $48 \mathrm{~s}$.

For this study we used a total of eight exposures in each of the two filters that covered the region near source D. Calibrated images were retrieved from the Hubble Data Archive, which had been processed using the latest NICMOS instrumental calibrations and data reduction software. The eight calibrated images in each filter were combined using the "multidrizzle" task in the STSDAS package of PyRAF. The final drizzle-combined images at $\mathrm{Pa} \alpha$ and $1.90 \mu \mathrm{m}$ were subsequently rescaled using the latest NICMOS photometric zero-point information to produce images in calibrated units of Jy.

To determine the position uncertainty of the stars, a Gaussian was fitted to two different F190N images with different exposures. Both images were used in making the drizzled mosaic. This allows us to measure random pointing errors from exposure to exposure, as well as the uncertainties in our measurements of the centroid in the images. The random errors in pointing and measurements are on the order of $\sim 0$ '. 1 . This is entirely consistent with the random relative errors associated with $H S T$ reacquiring guide stars in between exposures. In addition to this random error of $\sim 0$ '. 1 , there is also an absolute rms uncertainty of $\sim 0.2$ in how well the coordinates of the $H S T$ guide stars are known. Thus, the coordinates of the HST images can be trusted only at the level of 0 .'2. 
Table 1

2MASS, IRAC, and TEXES Flux Densities of Source D

\begin{tabular}{|c|c|c|c|}
\hline Source & Band & Magnitude & Flux Density (mJy) \\
\hline \multicolumn{4}{|l|}{ 2MASS 17455154-2900231 } \\
\hline & $J$ & $>17.607$ & $<0.1$ \\
\hline & $H$ & $>15.085$ & $<0.947$ \\
\hline & $K$ & $11.20 \pm 0.038$ & $22.08 \pm 0.7$ \\
\hline \multicolumn{4}{|l|}{ SSTGC $0556837\left(\right.$ GALCEN) ${ }^{\mathrm{a}}$} \\
\hline & 3.6 & $7.559 \pm 0.009$ & 266.0 \\
\hline & 4.5 & $5.571 \pm 0.004$ & 1062 \\
\hline & 5.8 & $4.152 \pm 0.006$ & 2511 \\
\hline & 8 & $3.617 \pm 0.006$ & 2292 \\
\hline \multicolumn{4}{|c|}{ SSTGLMA G359.9672-00.0811 (GLIMPSE II) } \\
\hline & 3.6 & $7.724 \pm 0.055$ & 228.4 \\
\hline & 4.5 & $5.755 \pm 0.071$ & 896.7 \\
\hline & 5.8 & $4.346 \pm 0.016$ & 2101 \\
\hline & 8 & $\ldots$ & $\ldots$ \\
\hline \multicolumn{4}{|l|}{ (IRACWORKS) } \\
\hline & 8 & $2.66 \pm 0.09$ & $5510 \pm 450$ \\
\hline \multicolumn{4}{|r|}{ 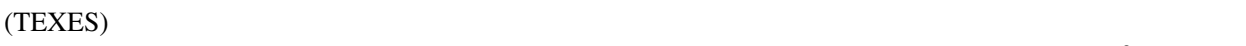 } \\
\hline & 12.8 & & $7500^{\mathrm{a}} \pm 1500$ \\
\hline
\end{tabular}

Note. ${ }^{\text {a }}$ May be affected by saturation.

\subsection{IRAC Measurements}

Infrared Array Camera (IRAC) observed the SGR A D as part of the Galactic center (GALCEN) survey by Stolovy et al. (2006). It was covered by five exposures with $1.2 \mathrm{~s}$ frame times. The IRAC flux densities for Sgr A D were obtained from both the GALCEN catalog (Ramirez et al. 2008) and the independently reduced GLIMPSE II catalog (an extension of the GLIMPSE survey, Benjamin et al. 2003). At 3.6, 4.5, and $5.8 \mu \mathrm{m}$ we use the GLIMPSE II measurements, which tend to be $\sim 15 \%$ lower than the GALCEN measurements. The GALCEN catalog indicates that the source is near or at saturation at all wavelengths, although at $3.6 \mu \mathrm{m}$ the source is only slightly past the nominal saturation limit. The GLIMPSE II catalog does not include an $8 \mu \mathrm{m}$ flux density. At this wavelength (and only this wavelength) the IRAC images show a local minimum at the peak of the source which is characteristic of a strongly saturated source. We have attempted to apply T. Jarret's IRACWORKS software ${ }^{10}$ to recover the flux of Sgr A D from the saturated images. At 3.6-5.8 $\mu \mathrm{m}$, IRACWORKS does not recognize Sgr A D as a saturated source, suggesting that cataloged flux densities are not less than $\sim 80 \%$ of the true values. At $8 \mu \mathrm{m}$, IRACWORKS successfully fits the source for only two out of five of the exposures, but does find a consistent result of $5510 \pm 450 \mathrm{mJy}$, which is about 2.5 times higher than the lower limit provided by the GALCEN catalog. Reported magnitudes and flux densities are listed in Table 1.

\section{RESULTS}

The large-scale distribution of thermal and nonthermal emission from the Galactic center is complex. However, within $4^{\prime}$ of Sgr A*, the distribution of dust, molecular, ionized, and synchrotron emission shows that the $50 \mathrm{~km} \mathrm{~s}^{-1}$ GMC M-0.02-0.07 interfaces with the SNR Sgr A East on one side and the cluster of $\mathrm{H}$ II regions on the opposite side. Figures 1(a) and (b) show the distributions of the 450 and $850 \mu \mathrm{m}$ thermal emission from dust, superimposed on a $20 \mathrm{~cm}$ radio continuum image, respectively

\footnotetext{
${ }^{10}$ http://ssc.spitzer.caltech.edu/dataanalysistools/tools/contributed/irac/ iracworks/
}

(Yusef-Zadeh \& Morris 1987; Tsuboi et al. 2009; Pierce-Price et al. 2000). Contours of submillimeter emission arises from the eastern edge of the $50 \mathrm{~km} \mathrm{~s}^{-1}$ molecular cloud. These figures show that the cluster of compact H II sources Sgr A A through $\mathrm{D}$ are distributed in a region relatively devoid of emission at 450 and $850 \mu \mathrm{m}$. The cavity in the submillimeter distribution might be caused by a drop in dust temperature. We made several cuts across the region where there is a depression of submillimeter emission and determined a constant $450 \mu \mathrm{m}$ to $850 \mu \mathrm{m}$ flux ratio. Since the emission is optically thin, this ratio corresponds to a constant temperature. We note that dust temperature cannot be constrained because it is entangled with the uncertainty in the ratio of optical depths. Since there is no evidence for a temperature gradient in the dust distribution, it is possible that the gas in the cavity is converted into formation of stars within the cloud. Source A is the most extended H II feature and lies to the north, whereas source $\mathrm{D}$, the most compact $\mathrm{H}$ II source, lies to the south. The distribution of the four $\mathrm{H}$ II regions appears to be anticorrelated with the distribution of dust and molecular gas, consistent with kinematic evidence that the $50 \mathrm{~km} \mathrm{~s}^{-1} \mathrm{GMC}$ is the parent cloud from which a cluster of massive young stars recently formed.

Figure 2(a) shows a grayscale image of free-free continuum emission from the Sgr A East $\mathrm{H}$ II cluster at $4.8 \mathrm{GHz}$ and Figure 2(b) shows the integrated [Ne II] line intensity map. The spatial distribution of [Ne II] line emission matches very closely the free-free $4.8 \mathrm{GHz}$ continuum distribution, demonstrating that $[\mathrm{Ne}$ II] is a good tracer of the ionized gas. Figure 2(c) shows a contour map of source D before and after deconvolution demonstrating the elongated structure of source $\mathrm{D}$ in both maps. Figure 2(d) shows the line and continuum maps in two different panels. The continuum map is from a $1200 \mathrm{~km} \mathrm{~s}^{-1}$ interval blueward of the line. The continuum map (right panel of Figure 2(d)) is narrower in the E-W direction than the line, and is centered on the peak of the line map within about 0.5 of the centroid of the line emission. Given the low resolution of the 2009 data, we cannot determine the relative position of the continuum and line peaks with respect to each other. The high-resolution data cannot detect the continuum emission. 

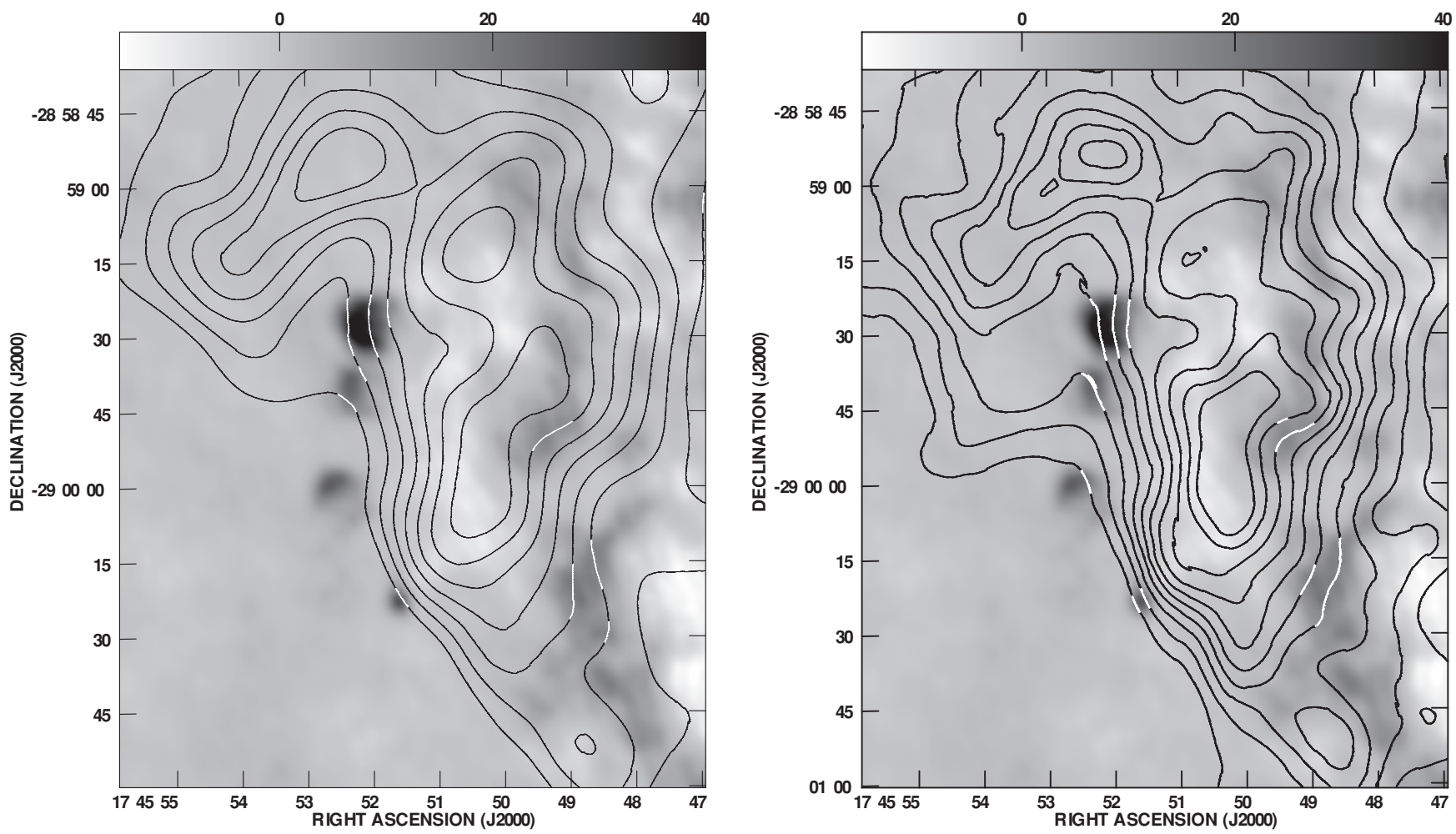

Figure 1. (a) Contours of $450 \mu \mathrm{m}$ emission from the Sgr A East $\mathrm{H}$ II region are superimposed on a $20 \mathrm{~cm}$ continuum image (black) and are represented at levels 32,34 , ..., $50 \mathrm{Jy}_{\text {beam }}^{-1}$ (beam size is $8^{\prime \prime} \times 8^{\prime \prime}$ ). (b) Similar to (a) except that contours of $850 \mu \mathrm{m}$ emission are presented at levels $(32,34, \ldots, 46) \times 0.2 \mathrm{Jy}$ beam ${ }^{-1}$ (beam size is $15^{\prime \prime} \times 15^{\prime \prime}$ ). The grayscale $20 \mathrm{~cm}$ continuum image (in black) has a resolution of $3 . .5$ and 2".9 (P.A. $=26^{\circ}$ ). The $450 \mu \mathrm{m}$ and $850 \mu \mathrm{m}$ emission trace dust emission from the $50 \mathrm{~km} \mathrm{~s}^{-1}$ molecular cloud GMC M-0.02-0.07. The background continuum emission has not been subtracted from submillimeter maps.

In this paper, we discuss two of the H II regions in the Sgr A East group: D, the bright, compact source to the south, and A, the most extended source to the north. Sources B and C have morphologies and kinematics similar to source A, but are more irregular and fainter.

\subsection{The D Source}

The brightest radio continuum source in Figure 2 is source $\mathrm{D}$, which is resolved for the first time into two equally bright radio continuum sources, D1 (East) and D2 (West), separated by $1^{\prime \prime} .2$ at position angle (P.A.) $\approx 70^{\circ}$. Each radio source consists of a compact source and a resolved structure elongated in the north-south (NS) direction. Contours of the emission at $4.87 \mathrm{GHz}$, as shown in Figure 3(a), extend for $\sim 18^{\prime \prime}$ or $\sim 0.7 \mathrm{pc}$, assuming that they are located at the Galactic center distance of $8 \mathrm{kpc}$. The Sgr A East $\mathrm{H}$ II regions have also been observed at 8.3 and $43 \mathrm{GHz}$. Figure $3(\mathrm{~b})$ shows a grayscale image of source $\mathrm{D}$ at $43 \mathrm{GHz}$ with a spatial resolution of $0.58 \times 00^{\prime \prime} 47$. Both sources D1 and D2 show N-S structure with an extent of $4^{\prime \prime}-5^{\prime \prime}$. The elongated features of sources D1 and D2 each show a compact component at $43 \mathrm{GHz}$. Table 2 gives the integrated flux, the size, and the peak flux of individual sources D1 and D2 at 4.87, 8.3, and $43.3 \mathrm{GHz}$, respectively. The Gaussian fitted positions of D1 and $\mathrm{D} 2$ are $\alpha, \delta(J 2000)=17^{\mathrm{h}} 45^{\mathrm{m}} 51^{\mathrm{s}} .62,-29^{\circ} 00^{\prime} 22^{\prime \prime} .59$ and $17^{\mathrm{h}} 45^{\mathrm{m}} 51^{\mathrm{s}} .53,-29^{\circ} 00^{\prime} 22^{\prime \prime} .82$, respectively. The images from which the fitted data are obtained have identical spatial resolution $0{ }^{\prime} 8 \times 0$ (' 4 at all three frequencies. The integrated fluxes $\mathrm{S}_{v}$ for sources D1 and D2 given similar spectral index of $\beta \sim 0.1$ (where $S_{v} \propto v^{\beta}$ ) between 8 and $44 \mathrm{GHz}$.

From the radio images, D1 and D2 appear to be a pair of stars, each of which has a NS bipolar jet. This situation could occur if each star has a disk collimating its jet, and the two disks are
Table 2

Parameters of the Fit to Source D G359.96-0.08

\begin{tabular}{lccccc}
\hline \hline Source & $v(\mathrm{GHz})$ & $S_{ \pm}^{\mathrm{a}} \sigma(\mathrm{mJy})$ & $\theta_{\text {maj }}{ }^{\prime}$ & $\theta_{\min }{ }^{\prime \prime}$ & $S(\mathrm{mJy})_{\text {peak }} \pm \sigma$ \\
\hline $\mathrm{D} 1$ & 4.87 & $41.4 \pm 1.1$ & 1.7 & 1.2 & $6.6 \pm 0.16$ \\
$"$ & 8.45 & $32.3 \pm 2.3$ & 1.7 & 0.7 & $8.6 \pm 0.5$ \\
$"$ & 44.34 & $38.9 \pm 3.6$ & 1.5 & 1.0 & $8.7 \pm 0.7$ \\
$\mathrm{D} 2$ & 4.87 & $46.2 \pm 1.1$ & 1.9 & 1.2 & $7.6 \pm 0.16$ \\
$"$ & 8.45 & $27.3 \pm 1.8$ & 1.4 & 0.5 & $10.0 \pm 0.5$ \\
$"$ & 44.34 & $29.4 \pm 2.2$ & 1.1 & 0.5 & $12.5 \pm 0.7$ \\
\hline
\end{tabular}

Note. ${ }^{\text {a }}$ The spatial resolution is $00^{\prime} 83$ and $0{ }^{\prime} .52\left(\right.$ P.A. $=11^{\circ}$ ).

coplanar, perhaps as a result of forming from the same cloud. But for this to be the right model, the two stars would have to be nearly identical in their luminosities and jet properties, including a difference in their $\mathrm{N}$ and $\mathrm{S}$ jets, as well as having closely aligned disks. This seems highly unlikely (see additional arguments against two stars, as described below). A model with one exciting source for the entire ionized gas distribution would seem more plausible if it could explain the spatial distribution of radio emission.

To examine the nature of the exciting star or stars in source D further, we compared the radio images with a Pa $\alpha$ and $1.90 \mu \mathrm{m}$ images of the region. The cross drawn in Figure 3(a) shows the peak position of the $1.90 \mu \mathrm{m}$ emission. The position of the stellar source does not change when both 1.90 and $1.87 \mu \mathrm{m}$ images are compared with each other. The centroids of the source at these wavelengths match to $00^{\prime} 02$. Since the images in the these two filters were obtained back to back, without moving the telescope, the relative positioning should be within 10-20 milli-arcsecond. The $1.90 \mu \mathrm{m}$ emission appears to coincide close to the compact component of source D2, suggesting that a single source in 



Figure 2. (a) $6 \mathrm{~cm}$ continuum image of the $\mathrm{Sgr} \mathrm{A}$ East $\mathrm{H}$ II region with a spatial resolution of $00^{\prime} 83$ and 0.52 (P.A. $=11^{\circ}$ ). The top three $\mathrm{H}$ II sources A-C are resolved having shell-like structures, whereas the most compact H II source D shows two compact sources elongated in the NS direction. (b) Same as (a) except that the map is based on integrated [Ne II] line emission over a velocity range of $V_{\mathrm{LSR}}=10-70 \mathrm{~km} \mathrm{~s}^{-1}$ corresponding to a spectral interval of $0.158 \mathrm{~cm}^{-1}$. Contours of the line emission are set quadratically at $(9,16,25, \ldots) \times 5 \times 10^{-4} \mathrm{erg} \mathrm{s}^{-1} \mathrm{~cm}^{-2} \mathrm{sr}^{-1}$. (c) Contour map of source $\mathrm{D}$ before and after deconvolution averaged over a $70 \mathrm{~km} \mathrm{~s}{ }^{-1}$ interval centered at $50 \mathrm{~km} \mathrm{~s}^{-1}$ with contour intervals $0.02 \mathrm{erg} \mathrm{s}^{-1} \mathrm{~cm}^{-2} \mathrm{sr}^{-1}$. (d) The line and continuum maps are shown in the left and right panels with contour intervals of $2.6 \times 10^{-3}$ and $3 \times 10^{-3} \mathrm{erg} \mathrm{s}^{-1} \mathrm{~cm}^{-2} \mathrm{sr}^{-1}$, respectively. The line map is the average over a $200 \mathrm{~km} \mathrm{~s}^{-1}$ interval centered on the line.

the vicinity of or coincident with D2 may be responsible for the ionization producing the extended radio continuum and $\mathrm{Pa} \alpha$ nebular emission. Alternatively, it is possible that the extinction is variable locally being responsible for a lack of $1.90 \mu \mathrm{m}$ detection from source D. In the continuum image the $\mathrm{D} 2$ source is very bright $5.1 \pm \mathrm{mJy}$, while $\mathrm{D} 1$ is nearly undetected $<0.9 \pm 0.1 \mathrm{mJy}$. In the emission-line image, on the other hand, $\mathrm{D} 1$ and D2 sources have the flux of $3.7 \pm 0.2$ and $7.8 \pm .2 \mathrm{mJy}$, respectively. If the extinction could be causing the D1 source to be hidden, then one would expect that both sources would be equally hidden in both bands, unless the $\mathrm{P} \alpha$ flux at the location of D1 is actually coming from something other than D1. If D1 were intrinsically as bright as D2 at $1.90 \mu \mathrm{m}$, but being hidden by extinction, then the ratio of their fluxes gives an equivalent magnitude difference of $1.9 \mathrm{mag}$ due to extinction. We can make the same analysis for the hypothetical D1 source using IRAC measurements. If a point source is present at D1, then its brightness must be at least 40 times lower than the source 

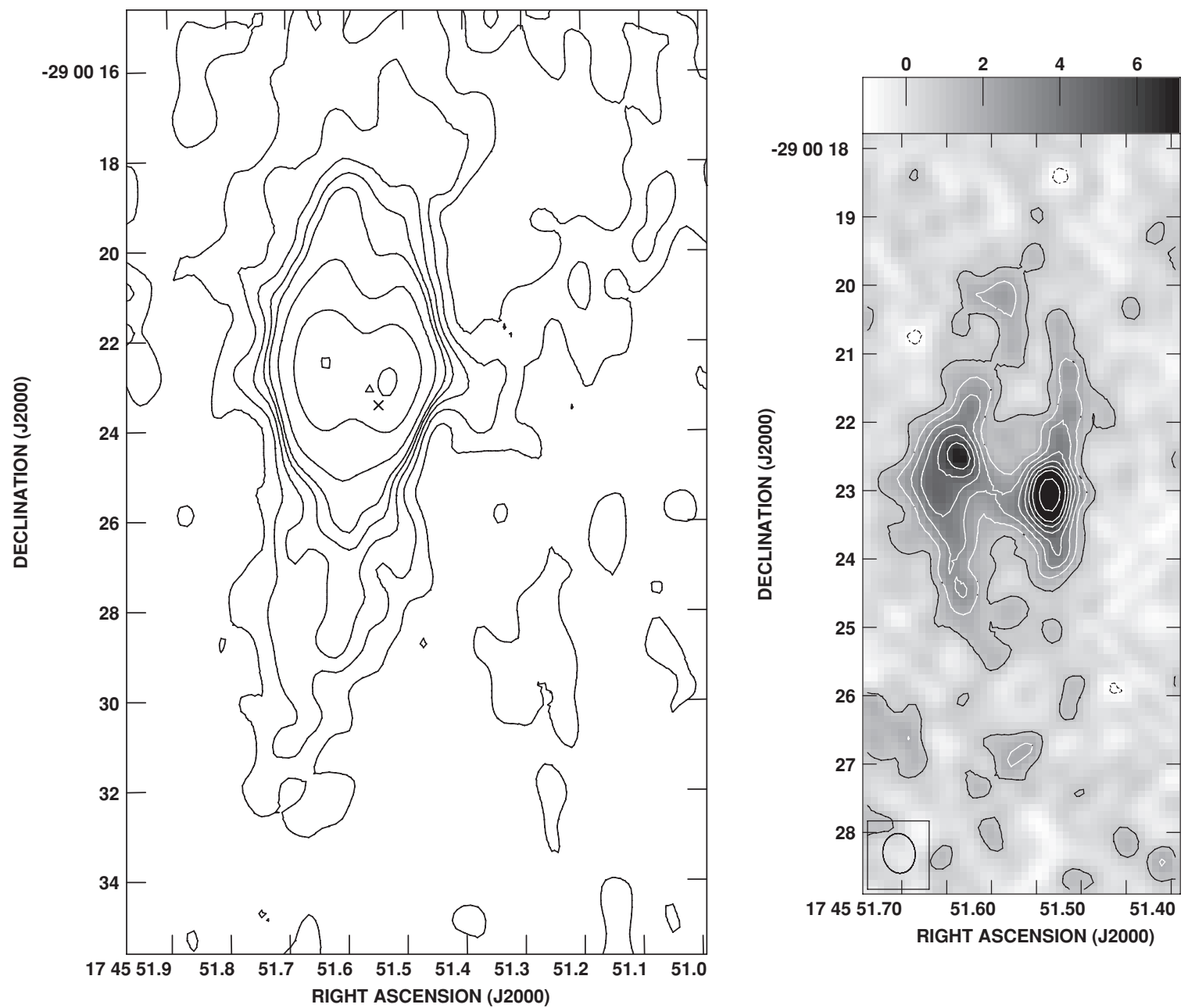

Figure 3. (a) Contours of $6 \mathrm{~cm}$ emission from source $\mathrm{D}$ are shown with levels set at $(1.25,1.5,1.75,2,2.25,3,6,15) \times 10^{-4} \mathrm{mJy}$ per beam. The cross and the triangle show the peak positions of the $1.90 \mu \mathrm{m}$ emission and the cataloged position of IRAC source with an uncertainty of $0.1-00^{\prime \prime} 2$, respectively. Both sources lie to the southeast of the peak radio emission from D2. (b) A grayscale image of source D at $43.3 \mathrm{GHz}$ with a spatial resolution of $00^{\prime \prime} 58$ and $0^{\prime \prime} .47\left(\mathrm{P} . \mathrm{A} .=8^{\circ}\right.$ ). Contours are set at $-1,1,2,3,4,5,6,7,8$, and 9 mJy per beam.

at D2. Otherwise, we would have detected the IRAC source looking slightly extended, and the position would be closer to the midpoint between D1 and D2. That means that a source at D1 must be at least 1.5-2.5 mag fainter than the source at D2.

In order to characterize the properties and the evolutionary phase of the stellar source responsible for the excitation of ionized material in source $\mathrm{D}$, we obtained the spectral energy distributions (SEDs) of the compact source closest to D2 using measurements from 2MASS, IRAC, and $\mathrm{Pa} \alpha$ and $1.90 \mu \mathrm{m}$ of NICMOS/HST from $1.24 \mu \mathrm{m}$ to $8 \mu \mathrm{m}$. The background subtracted Gaussian fitted position of the bright $1.90 \mu \mathrm{m}$ source gives the following coordinates: $\alpha, \delta(J 2000)=17^{\mathrm{h}} 45^{\mathrm{m}} 51^{\mathrm{s}} .545,-29^{\circ} 00^{\prime} 23^{\prime \prime} .420$. Similarly, the peak radio position D2 at $43 \mathrm{GHz}$ is at $\alpha, \delta(J 2000) 17^{\mathrm{h}} 45^{\mathrm{m}} 51^{\mathrm{s}} .536,-29^{\circ} 00^{\prime} 23^{\prime \prime} 064$. The $1.9 \mu \mathrm{m}$ fitted position gives an $\alpha, \delta$ offset of $\sim 0$ ' $^{\prime} 12$ and 0 ' 36 , respectively, southeast of the peak radio emission in D2. Given the random pointing errors and absolute uncertainty in the $1.9 \mu \mathrm{m}$ positions 0 '.2, it is likely that the $1.90 \mu \mathrm{m}$ source could lie between D1 and D2 but closer to D2 by 0'3 than to D1. Due to the poor resolution of the $12.8 \mu \mathrm{m}$ continuum data, we cannot identify the relative position of the continuum emission with respect to sources D1 and D2. Finally, the IRAC GALCEN and GLIMPSE II catalogs identify the position of the IRAC source as $\alpha, \delta(\mathrm{J} 2000) 17^{\mathrm{h}} 45^{\mathrm{m}} 51^{\mathrm{s}} .55,-29^{\circ} 00^{\prime} 23^{\prime \prime} .0$ with a $0^{\prime} .2$ and $0^{\prime} \cdot 3$ uncertainty, respectively. The centroid of the IRAC source is shown as a triangle with an uncertainty of 0.2 in Figure 3(a). This makes it very likely that the IRAC source corresponds to D2, and not D1. D1 and D2 could have not been separately resolved by IRAC, but if there were two sources of equal brightness, the point source would have appeared slightly more blurred and more centrally located than observed. Given the positional uncertainty, we cannot rule out the possibility of a single source that is centrally located between D1 and D2, but an association with D2 is most likely. Here, we name source D or G359.96-0.08 interchangeably between the central star and the peak of the radio emission.

Figure 4 shows IRAC images of the Sgr A East A-D regions at 3.6, 4.5, 5.8, and $8 \mu \mathrm{m}$ with Sgr A D source being saturated at $8 \mu \mathrm{m}$ (Stolovy et al. 2006; Ramirez et al. 2008; Arendt et al. 2008). The associated infrared dark cloud is best seen to the west of the $\mathrm{H}$ II regions where submillimeter emission is most prominent, as seen in Figure 1. D1 and D2 could have not been separately resolved by IRAC, but if there were two sources of equal brightness, the point source would appear slightly more blurred than observed. IRAC data indicate that the peak of the point source does seem to match closer to D2 than D1. We used lower limits to the saturated IRAC fluxes in the SED 


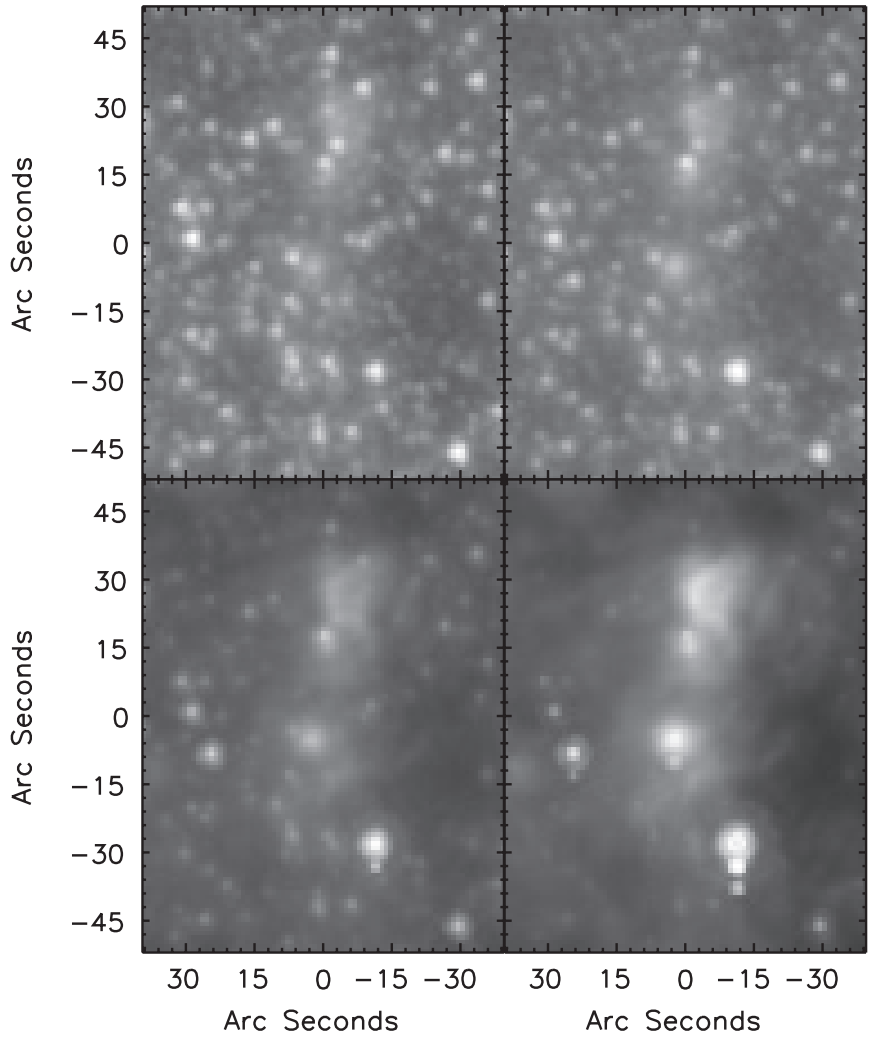

Figure 4. Spitzer IRAC images of the Sgr A East H II regions A-D. These logarithmically scaled images show emission at 3.6, 4.5, 5.8, and $8 \mu \mathrm{m}$ (left to right, top to bottom). The pixel size is $1^{\prime \prime}$. Bright sources at 5.8 and $8 \mu \mathrm{m}$ exhibit detector artifacts $5^{\prime \prime}$ and $10^{\prime \prime}$ to their south. This is especially distinct for Sgr A East D, which is the brightest source in the field and is obviously saturated at $8 \mu \mathrm{m}$

Table 3

Parameters of the SED Fit to G359.96-0.08

\begin{tabular}{lcccc}
\hline \hline Source & $\left\langle A_{\mathrm{v}}\right\rangle$ & $\left\langle M_{\star}\right\rangle$ & $\left\langle L_{\star}\right\rangle$ & $\left\langle T_{*}\right\rangle$ \\
\hline D2 & $42.9 \pm 5.8$ & $24.8 \pm 3.4$ & $8.1 \pm 2.6 \mathrm{E}+04$ & $3.6 \pm 0.6 \mathrm{E}+04$
\end{tabular}

fitting. The SED of compact component of D2 is analyzed by comparing to a set of SEDs produced by a large grid of young stellar object models (Robitaille et al. 2007; Whitney et al. 2003). A linear regression fitter was used to find all SEDs from the grid of models that are fit with the assumption that the difference between the $\chi^{2}$ per data point and the $\chi^{2}$ per data point of the best fit is less than three. The physical parameters corresponding to those models are averaged along with their standard deviations. Figure 5 shows the fit to the SED of the star associated with source D or G359.96-0.08, and Table 3 gives the mean values of visual extinction, stellar mass, luminosity, and temperature based on data from 2MASS, IRAC, $12.8 \mu \mathrm{m}$ continuum, and upper limits at 450 and $850 \mu \mathrm{m}$. These physical characteristics are consistent with the assertion that the central star of G359.96-0.08 is a hot, massive star. This star ionizes the surrounding gas, producing thermal radio continuum with a total integrated flux of 81 and $89 \mathrm{mJy}$ at 43.3 and $4.87 \mathrm{GHz}$, respectively. From the radio continuum and SED fitting using infrared continuum emission, it appears that the central star is of spectral type ranging between $\sim \mathrm{B} 0$ and $\mathrm{O} 9$ with a luminosity V class (Vacca et al. 1996) can account for the ionization of the gas in source D, including both D1 and D2.

Figure 6 shows four channel maps of [Ne II] line emission, each integrated over a velocity range of $15 \mathrm{~km} \mathrm{~s}^{-1}$. As is

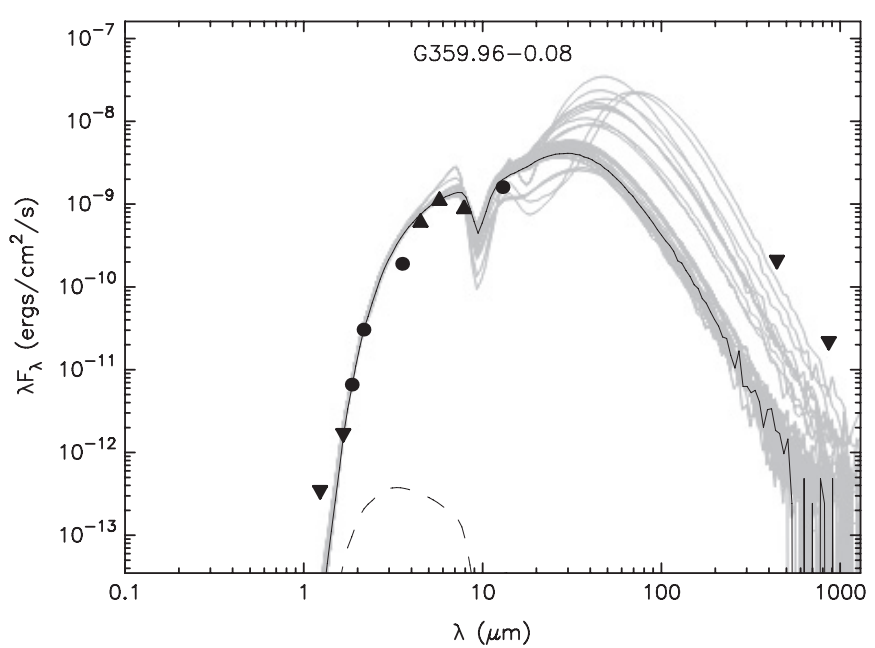

Figure 5. SED fits to source D. The closed circles are data points, downward facing triangle upper limit, upward facing triangles lower limits. The gray lines are all models that fit with $\chi^{2}<3$ higher than the best-fit model. The gray dashed line is the input stellar spectrum extincted by the best-fit foreground extinction.

true of the radio continuum distribution, the [Ne $\mathrm{II}]$ emission is symmetric about a NS line between D1 and D2, but only if the velocities are reflected about $v_{\mathrm{LSR}} \sim 47 \mathrm{~km} \mathrm{~s}^{-1}$. In an alternative display, the [Ne II] data cube is displayed in Figure 7 as a series of position-velocity (PV) diagrams. The panels in Figure 7 are cuts in right ascension at six different declinations, separated by $1^{\prime \prime}$. 1 . In the cut passing at declination of $-29^{\circ} 00^{\prime} 23^{\prime \prime} .3$, south of the peak emission, the line emission, as shown in the third panel from left, is narrow, both spectrally and spatially. The fourth panel from left shows clearly two blended peaks, one at $17^{\mathrm{h}} 45^{\mathrm{m}} 51^{\mathrm{s}} .60$, and one at 51.56. Following the naming used for the radio peaks, we will refer to them at D1 and D2. D1 peaks at $v_{\mathrm{LSR}}=48 \mathrm{~km} \mathrm{~s}^{-1}$, and has a red shoulder extending to $60 \mathrm{~km} \mathrm{~s}^{-1}$. D2 peaks at $45 \mathrm{~km} \mathrm{~s}^{-1}$, and has a blue shoulder extending to $35 \mathrm{~km} \mathrm{~s}^{-1}$. Going to the north, the spatial and spectral separation between D1 and D2 continues to increase, and the red and blue shoulders become more prominent. In the fifth panel from left, about $1^{\prime \prime} .5$ north of the peak, at $-29^{\circ} 00^{\prime} 21^{\prime \prime} .2$, the emission pattern forms an almost complete ring in the PV diagram. All of the PV diagrams show the same symmetry seen in the channel maps, being unchanged under a reflection through R.A. $=17^{\mathrm{h}} 45^{\mathrm{m}} 51^{\mathrm{s}} .58$, and $47 \mathrm{~km} \mathrm{~s}^{-1}$.

The strong symmetry of the ionized gas kinematics about a line between D1 and D2 makes it even less likely that these peaks could be ionized by two stars or that they could be ionized by a star centered on D2. It is possible that the ionizing star lies on the line of symmetry. However, the position of the star at $1.90 \mu \mathrm{m}$ shows that the source lies closer to D2 than D1.

\subsection{Sgr A East H II A-C}

Unlike the compact $\mathrm{H}$ II source D, sources A, B, and C appear rather similar, both morphologically, as seen in Figures 2(a) and (b), and kinematically, in the [Ne II] data cube.

Morphologically, source A appears to have cometary structure similar to that of G29.96-0.02 (e.g., Fey et al. 1995). To test this suggestion, Figure 8(a) shows the PV diagrams for sources A, B, and C. Sources B and C appear to have smaller extents than source A both spatially and kinematically. Sources A and $\mathrm{B}$ appear to have cometary structure, whereas source $\mathrm{C}$ is not 


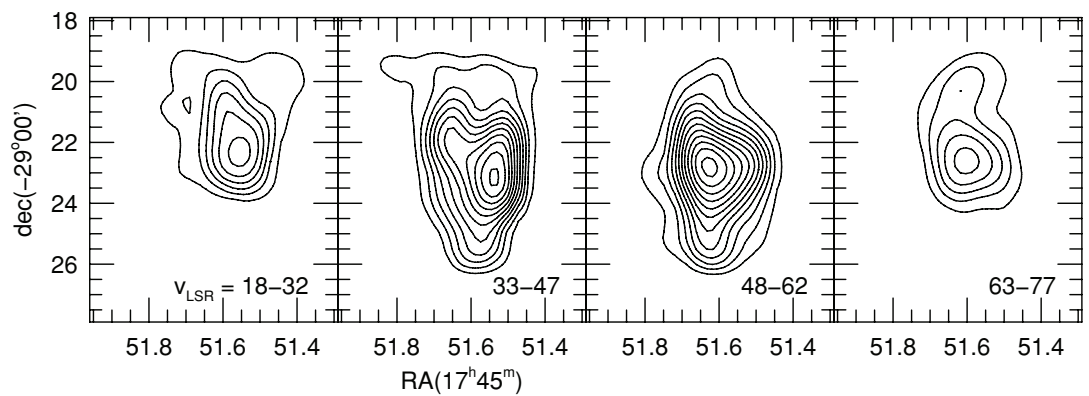

Figure 6. Four [Ne II] channel maps showing the distribution of ionized gas with contour levels set quadratically at $(9,16,25, \ldots) \times 3 \times 10^{-3} \mathrm{erg} \mathrm{s}^{-1} \mathrm{~cm}^{-2} \mathrm{~cm} \mathrm{sr}^{-1}$.

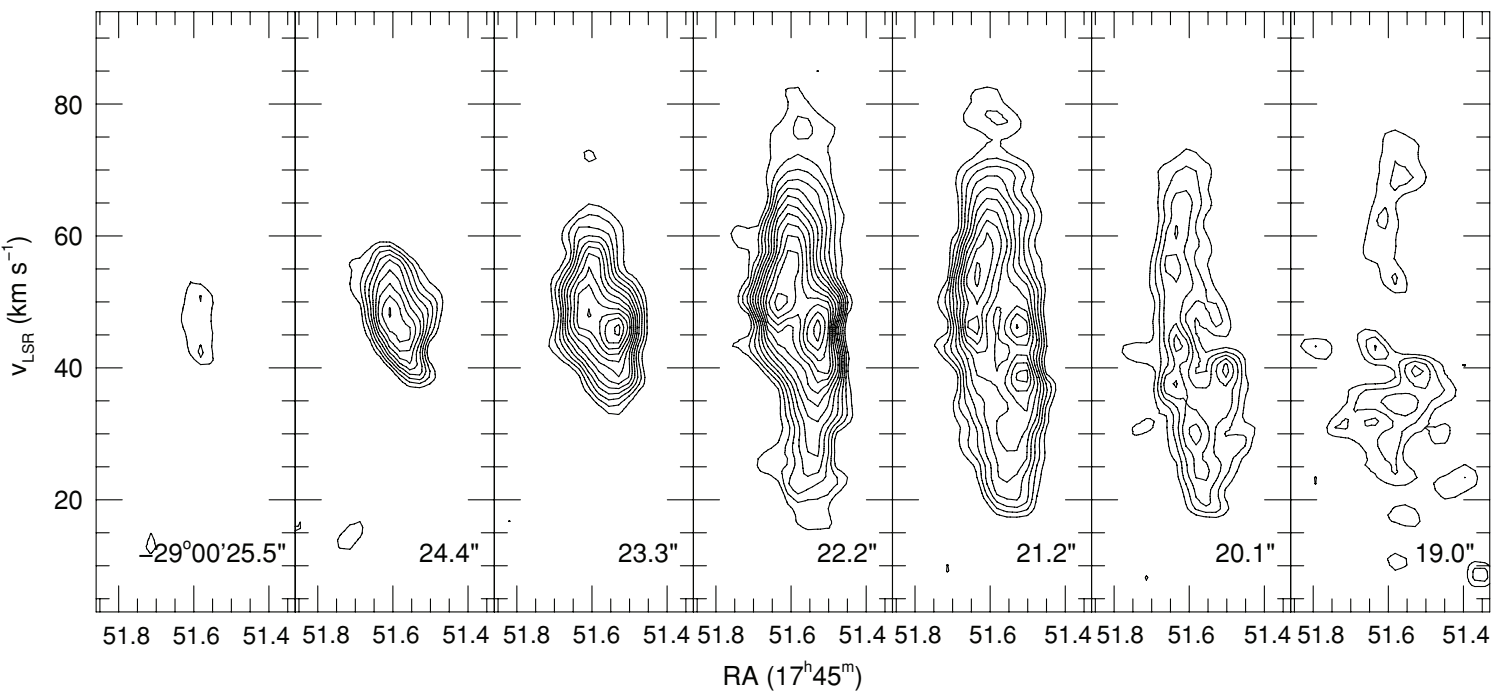

Figure 7. Contours of right ascension velocity diagrams of Sgr A D are shown in seven different declinations. The levels of integrated [Ne II] line intensity are set quadratically at $(9,16,25, \ldots) \times 1.9 \times 10^{-3} \mathrm{erg} \mathrm{s}^{-1} \mathrm{~cm}^{-2} \mathrm{~cm} \mathrm{sr}^{-1}$. The absolute coordinates of [Ne II] line images are done by comparing radio and [Ne II] images with each other.

resolved. The PV diagrams of R.A. cuts through $\mathrm{H}$ II source A at five different declinations are shown in Figure 8(b). The cuts along the northern and southern rims of the shell are narrower spectrally than the cuts passing through the center of the H II region, as shown in the middle panels. The broad lines in the eastern rim of the shell and double lines near the center of the shell show a pattern that is very similar to that seen in many UC H II regions (e.g., Zhu et al. 2008). The characteristic PV diagrams of several types of compact $\mathrm{H}_{\mathrm{II}}$ regions shown in Figures 45-48 of Zhu et al. show that the kinematics of source A is clearly cometary. PV diagrams of all three sources, with 3 " wide cuts, are shown in Figure 9. The PV diagram for source $\mathrm{B}$ is similar to that for A, but shows a smaller extent in both position and velocity, so is not so well resolved. Source C may be similar, but is even less well resolved.

The PV diagrams of Figure 8 match bow shocks in which the stellar wind of an $\mathrm{O}$ star sweeps the ionized gas into a thin, paraboloidal shell. The PV diagrams of sources B and C are very similar to that of source A. Most compact H II regions with recognizable kinematics observed in [Ne II] fall into the latter category, with the $\mathrm{H}_{\mathrm{II}}$ regions lying on the near sides of their molecular clouds, presumably because extinction through molecular clouds prevents observation of [Ne II] emission from those lying on the far sides. H II source A (as well as B and probably $\mathrm{C}$ ) is unusual in that the orientation of its PV diagram indicates that its head is tipped toward us. If it lies on the surface of a molecular cloud with its tail pointing out of the cloud, it must lie on the back side. This is somewhat surprising given the small extinction to the source. In addition, its velocity indicates that the star is moving relative to the $50 \mathrm{~km} \mathrm{~s}^{-1}$ molecular cloud. In fact, its kinematics matches very well that expected for ionized gas in a bow shock around an O star moving through molecular gas (see Wilkin 1996; Zhu et al. 2008; Arthur \& Hoare 2006). The star is moving to the east and toward us at an angle of roughly $30^{\circ}$. Perhaps this should not be surprising, given that this is the most commonly cited model for a cometary $\mathrm{H}$ II region, but it is not what was observed by Zhu et al., who found that most cometary $\mathrm{H}$ II regions are actually pressure-driven flows around stationary stars near the surfaces of molecular clouds. It seems likely that the turbulent environment near the Galactic center with high density gradient in molecular gas resulted in stars which rather quickly moved out of their natal clouds and are now drifting through lower density (and so lower extinction) molecular gas.

The ionizing stars for $\mathrm{A}, \mathrm{B}$, and $\mathrm{C}$ are not evident in IRAC data (see Figure 4). If present, they are not noticeably brighter than typical fore/background stars, nor are they located symmetrically at the centers of A-C. In stark contrast, D has an extremely bright point source. This difference alone indicates a significant difference between sources D and A-C. Additional difference between $\mathrm{D}$ and $\mathrm{A}-\mathrm{C} \mathrm{H}$ II regions comes from $M S X$ observations. Source D has the extremely bright point source that is increasingly more prominent with increasing wavelength. However, the relative brightness of $\mathrm{D}$ drops noticeably compared to $\mathrm{A}-\mathrm{C}$ when $M S X$ data at 8 and $24 \mu \mathrm{m}$ are used. This suggests a lower dust mass for $\mathrm{D}$, or that the bulk of the dust is warmer. 

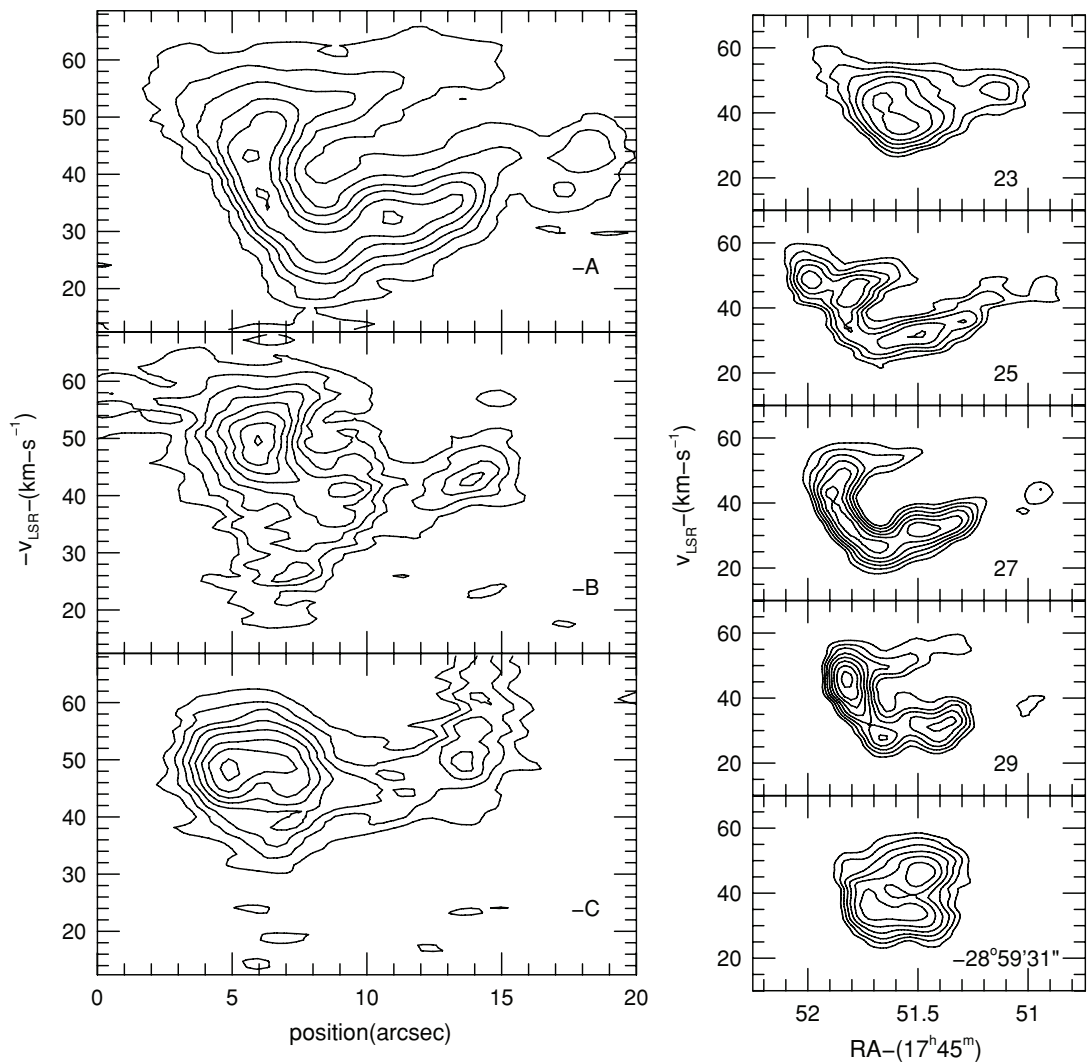

Figure 8. (a) PV diagrams for sources A, B, and C. For A and B, the cut runs EW (E at top of figure). For C it runs at $45^{\circ}$, NE-SW. All run near the centers of the sources, with $5^{\prime \prime}$ wide averaging bands. (b) Four [Ne II] PV diagrams of Sgr A East A source showing the velocity cuts at constant declination of $\left(-29^{\circ} 59^{\prime}\right)$ of $31^{\prime \prime} 2,29^{\prime \prime} 1,26^{\prime \prime} 9,24^{\prime \prime} 8$, and 22". 6 . Contour levels are set quadratically at $(9,16,25, \ldots) \times 2.7 \times 10^{-2} \mathrm{erg} \mathrm{s}^{-1} \mathrm{~cm}^{-2} \mathrm{~cm} \mathrm{sr}^{-1}$.

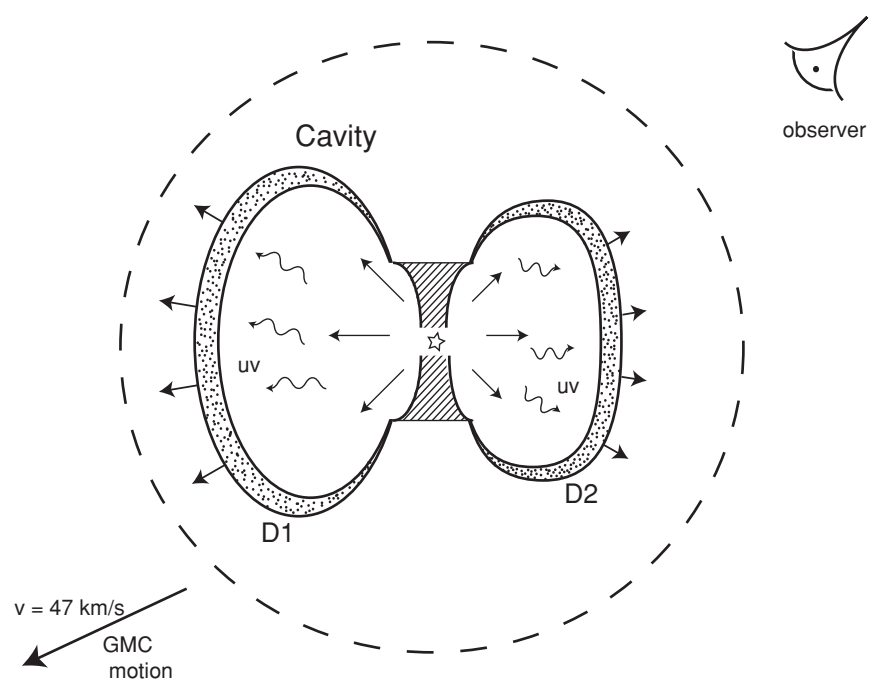

Figure 9. Schematic diagram of the features of the D source is drawn. The swept-up gas heated by UV radiation and the mass loss from the central young massive star and its disk are likely responsible for producing D1 and D1. The smaller size of D2 is considered to be due to inhomogeneity of the material surrounding the source.

\section{DISCUSSION}

\subsection{The Source of Ionization in Source D}

The radio measurements indicate that D1 is optically thin at $5-44 \mathrm{GHz}$, although the slight increase in flux from D2 at higher frequencies suggests that it may be partially optically thick at the very core. The $1.9 \mu \mathrm{m}$ measurements also indicate clearly a bright stellar source near the peak of the radio emission of D2. As the emission is predominantly optically thin in radio wavelengths, so adopting a distance $8 \mathrm{kpc}$ and an ionized gas temperature of $8000 \mathrm{~K}$, the total flux of $81 \mathrm{mJy}$ at $43.3 \mathrm{GHz}$ yields an estimate of the volume emission measure $\int n_{e}^{2} d V \approx$ $2 \times 10^{60} \mathrm{~cm}^{-3}$. The associated hydrogen recombination rate requires an ionization rate $N_{\mathrm{L}}=\alpha^{(2)} \int n_{e}^{2} d V \approx 6 \times 10^{47} \mathrm{~s}^{-1}$, where $\alpha^{(2)}$ is the hydrogen recombination coefficient into all levels but the ground state. As some ionizing photons might escape the immediate surroundings, be absorbed by dust, or be absorbed very close to the exciting star, where free-free optical depth may be large, this estimate is a lower limit on the ionizing photon production rate by the central source. The parameters of the SED of this source suggest that the stellar source G359.23-0.063 is associated with a $25 M_{\odot}$ star (see Table 3) and $8 \times 10^{4} L_{\odot}$. These parameters compare well with the $\approx(0.8-1.5) \times 10^{48} \mathrm{~s}^{-1}$ emitted by a O9/B0.5 star (Vacca et al. 1996). The full extent of the source in the NS direction is about $18^{\prime \prime}$. Assuming a source size of $9^{\prime \prime}$ and an expansion velocity of $\sim 30 \mathrm{~km} \mathrm{~s}^{-1}$ give an age of $\sim 10^{4}$ years. (This age estimate could be a lower limit if the motion is due to flows along the surfaces of the cloud.) This presents the earliest phase of massive star formation in the $50 \mathrm{~km} \mathrm{~s}^{-1}$ cloud.

The estimate for the mass of stars exciting sources A-C cannot be made as the stellar source is not identified in these $\mathrm{H}$ II regions. However, if we assume that the A-C sources have similar masses to that of $\mathrm{D}$, we can make an estimate of the total mass of stars in this regions. To compute the total cluster mass of Sgr A East, we adopt a standard broken power-law form of the initial mass function (Kroupa 2001) and assume $100 M_{\odot}$ in stars above $20 M_{\odot}$. This corresponds to a total stellar 
mass above $0.5 M_{\odot}$ of $940 M_{\odot}$ associated with a group of young stars in Sgr A East. Assuming that the typical age of the cluster is $\sim 10^{5}-10^{6}$ years, we estimate a star formation rate of $0.01-0.001 M_{\odot} \mathrm{yr}^{-1}$. We can also make an estimate of the total mass of molecular gas that went into forming stars. We used the $850 \mu \mathrm{m}$ images of Figure 1 to estimate the depleted gas mass by converting submillimeter flux to the mass of molecular gas. We estimate a subtracted flux of $15 \mathrm{Jy}$ at $850 \mu \mathrm{m}$ depleted from the region where the group of $\mathrm{H}$ II regions is distributed. We assumed that dust emission from the $50 \mathrm{~km} \mathrm{~s}^{-1}$ cloud was uniformly distributed inside and outside the $\mathrm{H}$ II regions prior the formation of stars. For a dust temperature of $20 \mathrm{~K}$ and a conversion factor from $850 \mu \mathrm{m}$ to gas mass (Pierce-Price et al. 2000), we find a rough total gas mass of $7.7 \times 10^{3} M_{\odot}$ that dissipated and converted into $940 M_{\odot}$ of stars.

The near-infrared observations show that despite the ionized gas morphology suggesting a pair of compact $\mathrm{H}$ II region, it is probably a single source that is exciting both D1 and D2. Presumably D1 is produced by irradiation of a dense clump of gas distributed within a cavity that has been partly evacuated of lower density gas by the stellar radiation field. In this scenario, the clump has an angular diameter of $~ 8000 \mathrm{AU}$ and lies at a projected distance of $\sim 12000$ AU from D2, and so intercepts approximately $10 \%$ of the ionizing photons escaping the D1 region. The observed $30 \mathrm{mJy}$ flux at $43 \mathrm{GHz}$ requires a total ionization rate at the clump surface of $\sim 2 \times 10^{47} \mathrm{~s}^{-1}$, implying that the exciting source is emitting ionizing photons at a rate $\sim 2 \times 10^{48} \mathrm{~s}^{-1}$.

\subsection{The Kinematics of Source D}

We now attempt to construct a model to explain the observations of source D. In particular, we need to explain the spatial distribution of the ionized gas and infrared continuum emission, as well as the motions of the ionized gas.

The ionized gas is distributed between two sources, D1 and $\mathrm{D} 2$, each of which is elongated in the NS direction. It is nearly symmetric about a NS line passing between D1 and D2, but with the eastern peak, D1, lying somewhat north of the western peak, D2, with a P.A. $\sim 70^{\circ}$. The infrared continuum, at both $1.9 \mu \mathrm{m}$ and $12.8 \mu \mathrm{m}$, shows a single peak that lies between the ionized gas peaks, but probably closer to D2. The kinematics of the ionized gas can be described by two probably related structures. A pair of spectrally broad features extend to the north from the D1 and D2 peaks. The eastern feature extends in velocity from near the molecular cloud velocity redward by about $30 \mathrm{~km} \mathrm{~s}^{-1}$. The western feature extends about $30 \mathrm{~km} \mathrm{~s}^{-1}$ blueward of the molecular cloud. Lower velocity emission is seen both north and south of the peaks, broadening both spectrally and spatially going to the north.

The two broad-lined emission features seem most naturally explained by a bipolar jet pair, probably interacting with the wall of a cavity. The jet could originate from a young star surrounded by a disk, lying between D1 and D2. The disk axis is tipped at a P.A. $\approx 70^{\circ}$, with the west side tipped toward us, so that the western jet is blueshifted. The fact that the jets are not seen extending to the east and west of D1 and D2 indicates that the observed [ $\mathrm{Ne} \mathrm{II}]$ emission is not from the jets themselves, but from the region of interaction between the jets and a surrounding wall. In fact, the bright emission peaks could be simply regions of a wall which are illuminated by ionizing radiation, but the width of the emission lines indicates that jets are needed. The offset between the position of the $1.9 \mu \mathrm{m}$ continuum and the position of the axis of the symmetry of D1/D2 can also be explained by inhomogeneity of gaseous material in the vicinity of the star and/or the motion of the star. Figure 9 shows a schematic diagram of the relative location of D1, D2, and the central massive star with a flaring disk. The fact that D1 is redshifted and D2 is blueshifted could be explained if the disk is seen at an angle between edge-on and face-on, with the west side of the disk tipped toward us. The lack of symmetry in this diagram is likely the result of density gradient surrounding the star. The $50 \mathrm{~km} \mathrm{~s}^{-1}$ molecular cloud lies mainly to the west of the central star.

High-mass stars efficiently photoevaporate their circumstellar disks on timescales of $10^{4}-10^{5}$ years. The piling up of the resulting disk wind as it expands against the surrounding medium could be responsible for the $\sim 0.1 M_{\odot}$ of ionized material we identify as sources D1 and D2. The semianalytic photoevaporated disk-wind model of Hollenbach et al. (1994) applied to a $28 M_{\odot}$ star yields a mass flux $\sim 3 \times 10^{-5} M_{\odot} \mathrm{yr}^{-1}$, indicating an age of $\sim 3 \times 10^{4}$ years. The momentum of the material would be maintained by contributions from the momentum fluxes $\dot{M} v \sim 3 \times 10^{-4} M_{\odot} \mathrm{km} \mathrm{s}^{-1}$ from each of the disk wind ( $v \gtrsim 10, \mathrm{~km} \mathrm{~s}^{-1}$; Drew et al. 1998; Hoare 2006) and stellar wind $\left(\sim 3 \times 10^{-7} M_{\odot} \mathrm{yr}^{-1}\right.$ and $\left.v \sim 1000 \mathrm{~km} \mathrm{~s}^{-1}\right)$. Another potential contribution could arise from a fraction of the total momentum flux $10^{5} L_{\odot} / c \sim 1 \times 10^{28} \sim 2 \times$ $10^{-3} M_{\odot} \mathrm{yr}^{-1} \mathrm{~km} \mathrm{~s}^{-1}$ in the photons emitted by the central star.

The extensions to the north could trace the regions of interaction of the jets with the cavity wall if the star is moving to the south, although it is not apparent why broad-lined emission would persist after the jets pass by. An explanation is also needed for the probable offset of the infrared continuum emission toward D2. One possibility is that the disk that collimates the jets is close enough to edge-on that it prevents a direct view of the central star even in the infrared. The near infrared continuum radiation could be scattered into our line of sight by the material that forms the ionized gas peak D2. Since D1 lies on the far side of the disk, backscattering (which would be less efficient) would be required for it D1 to be seen in scattered light. It may also be obscured by the disk if the disk extends out far enough to cover it. The $12.8 \mu \mathrm{m}$ continuum is unlikely to be scattered, but it could also be affected by extinction, and it is not so convincingly offset from the center of D as is the $1.9 \mu \mathrm{m}$ emission.

The spectrally narrow emission extending both north and south of the D peaks is even more difficult to explain than the broad-lined emission. The ring-like pattern seen in the PV diagrams in Figure 7 could be due to gas in each cut lying on an expanding ring. The fact that the PV rings increase in spatial and velocity extent going to the north would indicate that the three-dimensional ionized gas distribution is on the surface of an expanding cone, with the expansion speed increasing to the north. Alternatively, the gas could be flowing along the surface of a cone if it is accelerating so as to make its speed increase linearly with declination. However, we cannot propose a physical model that would cause either of these flow patterns. We know of no other case of an expanding cone like what we suggest or any reason a conical wall would expand in this way. A flow along the surface of a cone seems more natural, but all cometary $\mathrm{H}$ II regions we have observed, with sources $\mathrm{A}-\mathrm{C}$ being representative, are paraboloidal rather than conical, with much larger opening angles that in D. They also have most of their acceleration in a small distance from their vertexes. (A constant $d v / d t$ would result in $v^{2} \propto r$, or $d v / d r \propto r^{-1 / 2}$, rather than 
the observed constant $d v / d r$.) It is especially puzzling that the ionized cone has its vertex $3^{\prime \prime}$ south of $\mathrm{D}$, so presumably leading the ionizing star, which we think is moving in that direction. We have to conclude that we are unable to propose a consistent model for the NS velocity of ionized gas associated with source D. The narrow-lined gas appears to be distributed on the surface of a wedge or cone with an opening angle $\sim 20^{\circ}$, with its vertex $\sim 3^{\prime \prime}$ south of the star.

\subsection{The Kinematics of Source A}

Why are cometary $\mathrm{H}$ II sources $\mathrm{A}-\mathrm{C}$ oriented head-on, with velocity offsets indicating that their ionizing stars are moving through the $50 \mathrm{~km} \mathrm{~s}^{-1}$ molecular cloud, whereas most cometary $\mathrm{H}$ II regions seen in [ $\mathrm{Ne}$ II] are seen tail-on with little stellar motion? The answer may lie in the circumstances of their formation. The location of the Sgr A East H II regions near the edge of the Sgr A East SNR suggests that the ionizing stars may have formed as a result of compression of molecular gas by the SNR. If the stars formed in swept-up gas, they would have formed with the velocity of the gas. After their formation, the compressed shell would have slowed as more gas was swept up, and the stars could have drifted out of the compressed gas. The stars may now be moving through gas of low enough density that extinction does not affect the [Ne II] emission, as it would if they were still inside of a dense molecular cloud. From the orientation of its broken shell, it appears that the ionizing star of H II source A is moving to the east, as well as toward us. From the offset of its PV diagram, its motion relative to the $50 \mathrm{~km} \mathrm{~s}^{-1}$ molecular cloud is $\sim 30 \mathrm{~km} \mathrm{~s}^{-1}$. The ionizing stars of sources $\mathrm{B}$ and $\mathrm{C}$ have similar motions. In particular, the PV diagrams indicate that all three sources are moving toward us, while source $\mathrm{B}$ is moving to the east and source $\mathrm{C}$ is moving to the northeast.

The $\sim 50 \mathrm{~km} \mathrm{~s}^{-1}$ velocity width of the [NeII] line is consistent with other UC H II regions (e.g., Garay \& Lizano 1999). The characterization of a cluster of H II regions Sgr A A-D may not be correct, as these $\mathrm{H}$ II regions appear to be unbound due to their large physical separation, signifying isolated star formation near the Galactic center. Again, the site of on-going star formation is consistent with sites of massive star formation elsewhere (e.g., Garay \& Lizano 1999).

\subsection{Conclusions}

We thank the referee for a thorough reading of the paper and for useful comments. We have presented multi-wavelength observations of a chain of $\mathrm{H}$ II regions located at the eastern edge of the Sgr A East SNR and the $50 \mathrm{~km} \mathrm{~s}^{-1}$ molecular cloud. These HiI regions show the youngest star formation activity closest to the Galactic center. The youngest member of Sgr A East H II regions G359.956-0.08 or the Sgr A D source is estimated to have an age of roughly $1.5 \times 10^{4}$ years and consists of two elongated ionized features. We identified the central star responsible for ionizing both components of the $\mathrm{D}$ source. The SED fit of the central star indicates a mass of $25 M_{\odot}$ and a luminosity of $8 \times 10^{4} L_{\odot}$. The kinematics of ionized gas show an E-W red- and blueshifted flow with respect to the central star and a complex asymmetric velocity structure in the N-S direction. We presented a simple model in which the central star is surrounded by a disk constraining the flow of ionized wind. In this picture, the UV radiation from the central hot star may generate an outflow from the disk, sweeping up the interstellar medium gas and forming the two components of ionized gas D1 and D2. Given that high density molecular material is distributed to the west, the ionized feature D2 is expected to lie closer to the central star. Future high spectral resolution observations of ionized gas and detailed modeling of the complex $\mathrm{N}-\mathrm{S}$ velocity structure should explain better the kinematics of ionized gas presented here.

This work is partially supported by grants AST-0807400 (to F.Y.Z.) and AST-0607312 (to J.H.L.) from the National Science Foundation. This research has made use of the NASA/IPAC Infrared Science Archive, which is operated by the Jet Propulsion Laboratory, California Institute of Technology, under contract with the National Aeronautics and Space Administration.

\section{REFERENCES}

Arendt, R. G., et al. 2008, ApJ, 682, 384

Arthur, S. J., \& Hoare, M. G. 2006, ApJS, 165, 283

Bartko, H., et al. 2009, ApJ, 697, 1741

Benjamin, R. A., et al. 2003, PASP, 115, 953

Cotera, A. S., Simpson, J. P., Erickson, E. F., Colgan, S. W. J., Burton, M. G., \& Allen, D. A. 1999, ApJ, 510, 747

dePree, C. G., Goss, W. M., \& Gaume, R. A. 1998, ApJ, 500, 847

Drew, J. E., Proga, D., \& Stone, J. M. 1998, MNRAS, 296, L6

Fey, A. L., Gaume, R. A., Claussen, M. J., \& Vrba, F. J. 1995, ApJ, 453, 308

Figer, D. F., et al. 2002, ApJ, 581, 258

Garay, G., \& Lizano, S. 1999, PASP, 111, 1049

Ghez, A. M., et al. 2008, ApJ, 689, 1044

Gillesen, S., Eisenhauer, F., Trippe, S., Alexander, T., Genzel, R., Martins, F., \& Ott, T. 2009, ApJ, 692, 1075

Goss, W. M., Schwarz, U. J., van Gorkom, J. H., \& Ekers, R. D. 1985, MNRAS, 215,69

Hoare, M. G. 2006, ApJ, 649, 856

Hoare, M. G., Kurtz, S. E., Lizano, S., Keto, E., \& Hofner, P. 2007, in Protostars and Planets V, ed. B. Reipurth, D. Jewitt, \& K. Keil (Tucson, AZ: Univ. Arizona Press), 181

Hollenbach, D., Johnstone, D., Lizano, S., \& Shu, F. 1994, ApJ, 428, 654

Kroupa, P. 2001, MNRAS, 322, 231

Lacy, J. H., Richter, M. J., Greathouse, T. K., Jaffe, D. T., \& Zhu, Q. 2002, PASP, 114,153

McKee, C. F., \& Ostriker, E. C. 2007, ARA\&A, 45, 565

Morris, M., \& Serabyn, E. 1996, ARA\&A, 34, 645

Pierce-Price, D., et al. 2000, ApJ, 545, L121

Ramirez, S. V., Arendt, R. G., Sellgren, K., Stolovy, S. R., Cotera, A., Smith, H. A., \& Yusef-Zadeh, F. 2008, ApJS, 175, 147

Reid, M. J., \& Brunthaler, A. 2004, ApJ, 616, 872

Robitaille, T. P., Whitney, B. A., Indebetouw, R., \& Wood, K. 2007, ApJS, 169, 328

Serabyn, E., Lacy, J. H., \& Achtermann, J. M. 1992, ApJ, 395, 166

Shu, F. H., Adams, F. C., \& Lizano, S. 1987, ARA\&A, 25, 23

Stolovy, S., et al. 2006, J. Phys. Conf. Ser., 54, 176

Tsuboi, M., Miyazaki, A., \& Okumura, S. K. 2009, PASJ, 61, 29

Vacca, W. D., Garmany, C. D., \& Shull, J. M. 1996, ApJ, 460, 914

Wang, Q. D., et al. 2010, MNRAS, 402, 895

Whitney, B. A., Wood, K., Bjorkman, J. E., \& Cohen, M. 2003, ApJ, 598, 1079

Wilkin, F. P. 1996, ApJ, 459, L31

Yusef-Zadeh, F., \& Morris, M. 1987, ApJ, 320, 545

Yusef-Zadeh, F., Telesco, C. M., \& Decher, R. 1989, in IAU Symp. 136, The Center of the Galaxy, ed. Mark Morris (Dordrecht: Kluwer), 287

Yusef-Zadeh, F., \& Wardle, M. 1993, ApJ, 405, 584

Zhu, Q.-F., Lacy, J. H., Jaffe, D. T., Richter, M. J., \& Greathouse, T. K. 2008, ApJS, 177, 584

Zinnecker, H., \& Yorke, H. W. 2007, ARA\&A, 45, 481 